\title{
DA GRANDE MODERAÇÃO A GRANDE RECESSÃO: ALGUMAS CONSIDERAÇÕES ACERCA DA CONSTRUÇÃO E REVISÃO DO NOVO CONSENSO MACROECONÔMICO A PARTIR DA CRISE DE 2007-2008
}

\author{
Paulo Saraiva ${ }^{1}$ \\ Luiz Fernando de Paula ${ }^{2}$ \\ André de Melo Modenesi ${ }^{3}$
}

Resumo: As últimas quatro décadas de pensamento econômico foram marcadas pela ascensão, hegemonia e revisão do liberalismo econômico compendiado no Novo Consenso Macroeconômico. A consolidação de uma "nova síntese neoclássica" no início dos anos 1990 resultou na implantação do regime de metas de inflação em diversos países, na defesa de uma grande moderação no início dos anos 2000 e na ocorrência da crise financeira internacional a partir de 2007. Contudo, a revisão do Novo Consenso Macroeconômico, ainda em curso e com diversos dissensos, vem relativizando o impacto da crise e propondo ajustes às políticas econômicas apesar de reafirmar os fundamentos teóricos da ortodoxia.

Palavras-chave: Novo consenso macroeconômico. Revisão do novo consenso macroeconômico. A grande moderação. Crise financeira. Regime de metas de inflação.

\section{FROM GREAT MODERATION TO GREAT RECESSION: SOME CONSIDERATIONS ABOUT THE CONSTRUCTION AND REVISION OF THE NEW CONSENSUS MACROECONOMICS AFTER THE 2007-2008 CRISIS}

Abstract: The last four decades of economic thought have been marked by the rise, hegemony and revision of economic liberalism epitomized by the New Consensus Macroeconomics. The consolidation of a "new neoclassical synthesis" in the early 1990s resulted in the implementation of inflation targeting regime in several countries, in the defense of great moderation in the early 2000s and the occurrence of the financial crisis from 2007. However, the review of the New Macroeconomic Consensus, still ongoing and with many disagreements comes relativizing the impact of the crisis and proposing adjustments to economic policies despite of reaffirming the orthodoxy theoretical foundations.

Keywords: New consensus macroeconomics. New consensus macroeconomics review. The great moderation. Financial crisis. Inflation target.

\section{DE LA GRAN MODERACIÓN A LA GRAN RECESIÓN: ALGUNAS CONSIDERACIONES ACERCA DE LA CONSTRUCCIÓN Y REVISIÓN DEL}

\footnotetext{
${ }^{1}$ Professor Adjunto da Universidade Federal Rural do Rio de Janeiro (UFRRJ), pjsaraiva@terra.com.br, https://orcid.org/0000-0003-3414-7337

2 Professor do Instituto de Economia da Universidade Federal do Rio de Janeiro (IE/UFRJ), Professor Voluntário do Instituto de Estudos Políticos e Sociais (IESP/UERJ) e Pesquisador do CNPq, luizfpaula@terra.com.br, https://orcid.org/0000-0001-9770-516X.

${ }^{3}$ Professor Associado do Instituto de Economia da Universidade Federal do Rio de Janeiro (IE/UFRJ) e Pesquisador do CNPq, amodenesi@gmail.com, https://orcid.org/0000-0001-5392-2920
} 


\section{NUEVO CONSENSO MACROECONÓMICO A PARTIR DE LA CRISIS DE 2007- 2008}

Resumen: Las últimas cuatro décadas de pensamiento económico fueron marcadas por la ascensión, hegemonía y revisión del liberalismo económico compendiado en el Nuevo Consenso Macroeconómico. La consolidación de una "nueva síntesis neoclásica" a principios de los años 1990 resultó en la implantación del régimen de metas de inflación en diversos países, en la defensa de una gran moderación a principios de los años 2000 y en la ocurrencia de la crisis financiera internacional a partir de 2007. Sin embargo, la revisión del Nuevo Consenso Macroeconómico, aún en curso y con diversos disensos, viene relativizando el impacto de la crisis y proponiendo ajustes a las políticas económicas a pesar de reafirmar los fundamentos teóricos de la ortodoxia.

Palabras clave: Nuevo consenso macroeconómico. Revisión del nuevo consenso macroeconómico. La gran moderación. Crisis financiera. Régimen de metas de inflación.

\section{INTRODUÇÃO}

O debate em relação ao Novo Consenso Macroeconômico (NCM), ou o que alguns autores denominaram de Nova Síntese Neoclássica, ou ainda de Nova Economia Keynesiana, envolve um conjunto extenso e interligado de questões teóricas, estudos empíricos e de experiências práticas de diversos Bancos Centrais (BC), desde o final da década de 80, em diversos países desenvolvidos e em desenvolvimento. A pluralidade de definições deve-se, em boa medida, a incorporação pela corrente Novo-Keynesiana, de um amplo desenvolvimento teórico e metodológico, desde a década de sessenta, que colocaram em questionamento a validade da teoria e política econômica keynesiana.

O núcleo deste consenso é composto por contribuições dos monetaristas, novo-clássicos, dos modelos de ciclo real de negócios, bem como pela própria corrente Novo-Keynesiana. A busca por uma nova síntese, gerou um papel residual a política fiscal e financeira em detrimento da política monetária (PM), reafirmação da neutralidade da moeda no longo prazo, a incorporação de expectativas racionais, aceitação da crítica de Lucas, da hipótese de mercados eficientes e dos modelos dinâmicos de equilíbrio geral estocástico. No entanto, mercados de concorrência imperfeita e fricções de preços e salários foram adicionados ao corpo teórico, assim como a regra de Taylor substituiu a função de equilíbrio do mercado monetário (LM), 
tornando-se um importante instrumento da PM, conjuntamente com a gestão das expectativas.

O resultado desta síntese foi à consolidação do Regime de Metas de Inflação (RMI), que representou um conjunto de orientações teóricas de como a política econômica, dirigida pela PM deveria ser conduzida por um BC. MISHKIN (2007) chegou a afirmar que se estava construindo uma "ciência monetária", enquanto BERNANKE (2004) atribuiu a esta estabilidade econômica (inflação e desemprego) das últimas décadas o qual denominou de "a grande moderação". No entanto, a crise americana iniciada em 2007 e agonizada a partir do segundo semestre de 2008, demonstrou que durante um período de aparente estabilidade, emergia um processo de ampliação da alavancagem e do nível de exposição ao risco no mercado financeiro americano, que acabou por gerar uma crise financeira internacional e, na sequência, uma "grande recessão".

$\mathrm{Na}$ contramão da calmaria vinha se desenvolvendo um movimento especulativo nos mercados financeiros americanos, particularmente nos mercados de hipoteca, cuja inter-relação com os demais mercados gerou uma crise sistêmica e internacional com proporções similares a crise de 1929. Como resultado imediato, medidas não convencionais de política econômica (monetárias e fiscais) foram necessárias e implantadas em grande magnitude para amenizar e reverter os impactos da ruptura financeira e da recessão na atividade economia ${ }^{4}$.

A partir de então, os fundamentos teóricos e as recomendações de política econômica do NCM vem sendo questionados, tanto no ambiente acadêmico, quando na esfera política, exigindo que seus proponentes e defensores, fornecessem respostas sobre as limitações e deficiências do modelo de política econômica adotada ao longo das últimas décadas. O objetivo deste artigo é mapear esta discussão sobre a revisão do NCM realizada no pós-crise de 2008 pela ortodoxia econômica nos EUA ${ }^{5}$. Nesta perspectiva, o artigo está estruturado em 3 seções, além desta introdução. Na seção 2 é apresentada a fundamentação teórica do NCM, um modelo estilizado e a sua operacionalização por meio do RMI. Na seção 3 avalia-se os aspectos centrais da revisão do NCM. Por fim, a seção 4 conclui o artigo.

\footnotetext{
${ }^{4}$ Para uma análise das políticas monetárias não-convencionais adotadas nos EUA a partir de 2007, ver Saraiva et al (2017).

5 Para uma crítica externa e heterodoxa a revisão do NCM, ver Paula et al (2018).
} 


\title{
A GRANDE MODERAÇÃO, O NCM E O RMI
}

A economia americana e diversos países ${ }^{6}$, ao longo das décadas de 1980 , 1990 e até meados de 2000, experimentaram um período de redução na variabilidade do produto e da inflação. Nos EUA, a volatilidade do produto teve uma redução de $50 \%$, ao passo que a volatilidade da inflação recuou cerca de $2 / 3$. Esse fenômeno foi chamado de a "grande moderação", sendo atribuída, em boa medida, ao êxito da $\mathrm{PM}^{7}$. Neste período, um dos avanços mais significativos, foi a compreensão de que os choques de demanda e o seu impacto sobre a produção e a inflação podem ser estabilizados pela PM, por meio de uma regra de Taylor (1993), ao passo que choques de oferta, exigem da autoridade monetária a escolha entre estabilizar o produto ou a inflação (BERNANKE, 2004).

Os defensores de uma inflação baixa e estável argumentam que a volatilidade, assim como uma taxa de crescimento mais elevada da inflação de uma economia, provoca maiores incertezas presentes e futuras, devido a uma maior variabilidade nos preços relativos e na taxa de juros real da economia, causando distorções nas decisões das famílias, em termos de consumo, e das empresas, no que se refere aos investimentos em bens de capital e estoque de produtos, promovendo ineficiência no sistema econômico. Ademais a inflação representa um imposto sobre a retenção de moeda (como meio de troca), repercutindo em um desvio dos investimentos empresariais para as aplicações financeiras. Por outro lado, uma inflação demasiadamente baixa tem implicações importantes em regimes monetários que utilizam como instrumento de $\mathrm{PM}$, a taxa de juros nominal de curto prazo, dado o limite inferior igual a zero (BERNANKE, 2004; MISHKIN, 2007 e GOODFRIEND, 2007). Segundo BERNANKE (2005, p. 277):

\begin{abstract}
"As it turned out, the low-inflation era of the past two decades has seen not only significant improvements in economic growth and productivity but also a marked reduction in economic volatility, both in the United States and abroad, a phenomenon that has been dubbed "the Great Moderation." Recessions have become less frequent and milder, and quarter-to-quarter volatility in output and employment has declined significantly as well. The sources of the Great Moderation remain somewhat controversial, but, as I have argued elsewhere, there is evidence for the view that improved control of inflation has contributed in important measure to this welcome change in the economy".
\end{abstract}

6. Refere-se aos países que adoram o RMI ou alguma variante deste regime, tais como a Inglaterra, Nova-Zelândia, Canadá, Japão, dentre outros.

7. Outras explicações foram as mudanças estruturais e a "boa sorte". Para uma discussão detalhada ver Bernanke (2004). 
A representação simplificada em defesa dos benefícios de uma PM eficiente, pode ser visualizada no gráfico 1, a partir de uma Curva de Taylor que mostra as combinações entre a volatilidade do produto e da inflação. Nesta, a PM que almeja bem-estar social poderá escolher uma combinação entre variabilidade do produto e da inflação, representado pela curva $\mathrm{TC}_{2}$. Esta retrata como o trade-off da $\mathrm{PM}$, durante os anos 60 e 70, estava distanciado do ideal - acima da fronteira eficiente, representada por $\mathrm{TC}_{1}$ - gerando uma combinação mais elevada, entre a variabilidade do produto e da inflação $\left(\mathrm{TC}_{2}\right)$. No entanto, a explicação para o período de "grande moderação" deriva da adoção de uma PM mais eficiente - Regra de Taylor, a partir da década de 1990, gerando redução da variabilidade entre o produto e a inflação, e pode ser observada pelo deslocamento da curva $\mathrm{TC}_{2}$ para $\mathrm{TC}_{1}$ (BERNANKE, 2004).

\section{Gráfico 1. PM e variabilidade do produto e da inflação}

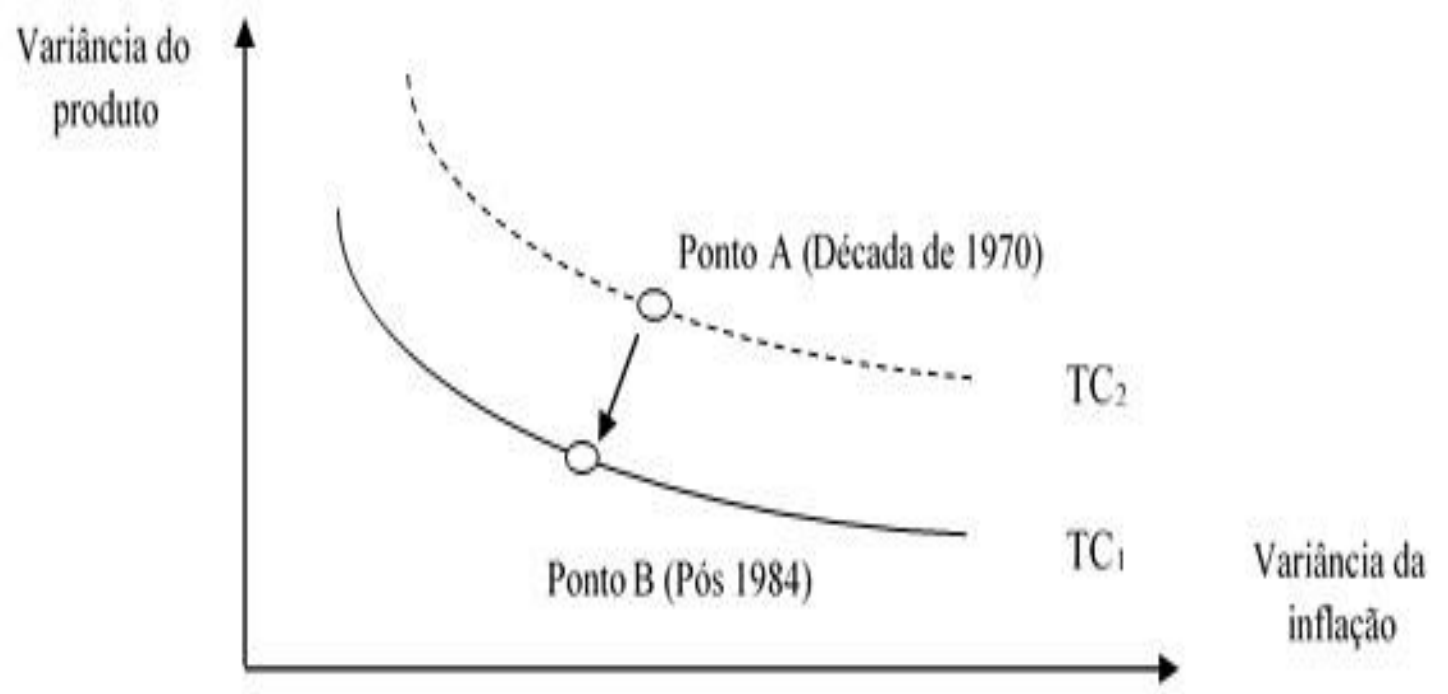

Fonte: Bernanke (2004)

As justificativas, segundo BERNANKE (2004), para o sucesso logrado pela PM, desde outubro de 1979 nos EUA, podem ser atribuídas a quatro fatores, quais sejam: i) As mudanças na PM objetivaram a redução e estabilização da inflação, promovendo mudanças estruturais no regime de PM; ii) As mudanças na PM reduziram o tamanho e a frequência dos choques na economia; iii) $A$ PM, ao afetar os choques da economia, altera a sensibilidade dos preços e decisões econômicas dos agentes; e iv) As expectativas de inflação desempenham um papel importante, e tem forte impacto sobre o nível de preços, tendo sido influenciadas pelo novo regime monetário adotado. 
Segundo BERNANKE (2004), as evidências de uma "grande moderação" reforçaram a eficiência do regime de política macroeconômica que vem sendo adotadas por diversos países ao longo das últimas três décadas. Este representa a visão do NCM, que vem sendo operacionalizado por diversos países desenvolvidos e emergentes através de um RMI, ou uma variante deste modelo.

\section{Vigamentos}

O NCM surge da conjugação de elementos teóricos e empíricos oriundos das escolas monetarista, novo-clássica, do ciclo real de negócios e novo-keynesiana, e representa um movimento de convergência entre diferentes escolas, similar ao promovido por Paul Samuelson na década de 1940 na chamada síntese neoclássica. Seus aspectos centrais são a incorporação da hipótese de expectativas racionais, a relevância da demanda agregada na condução da atividade econômica no curto prazo, devido à presença de rigidez de preços e salários microfundamentada, além da importância das imperfeições de mercado para a explicação da trajetória da economia em relação à taxa de crescimento no longo prazo.

Enquanto que Friedman estabeleceu as bases do monetarismo, a partir do conceito de taxa natural de desemprego e curva de Phillips aceleracionista, e ainda do restabelecimento da "nova" teoria quantitativa da moeda, coube a LUCAS (1972) estabelecer as bases da teoria de expectativas racionais, segundo o qual os agentes formam expectativas de modo a evitar erros sistemáticos. Essa abordagem teve forte impacto teórico e subsidiou políticas desinflacionarias nos EUA através da proposição de que a política monetária (PM) pode controlar sozinha a inflação sem a necessidade da utilização de outros instrumentos. A revolução das expectativas racionais implicou que as expectativas dos agentes sobre o comportamento da PM exercem impacto sobre o produto de uma econômica.

No final da década de 1970, a discussão apresentada por KYDLAND e PRESCOTT (1977), em relação à política de regra versus discrição e o problema da inconsistência temporal da PM, torna-se seminal. Os autores argumentam que os resultados econômicos poderão melhorar se as políticas econômicas tiverem um compromisso crível, ao passo que a PM conduzida de forma discricionária estará sujeita ao problema de inconsistência temporal. No entanto, estes autores não desenvolveram como o Banco Central $(\mathrm{BC})$ deve obter a credibilidade. Neste ponto, 
ROGOFF (1985) desenvolve a proposição analítica de um presidente do Banco Central que tenha um grau de aversão à inflação maior do que a média do público em geral, que, contudo, pode não ser suficiente, dado que as pressões políticas e da sociedade podem influenciar a condução da PM, necessitando, portanto, da percepção por parte dos agentes da independência do BC. Já BARRO e GORDON (1983) discutiram a importância da reputação do BC como um elemento central para construção da credibilidade.

Na década de 1980, KYDLAND e PRESCOTT (1982) desenvolvem o modelo de ciclo real de negócios ( $\mathrm{CRN}$ ), enfatizando o papel das variáveis reais, em particular os choques tecnológicos, na explicação das flutuações econômicas. Este modelo e os seus desenvolvimentos posteriores dominaram a pesquisa na área macroeconômica até o início da década de 1990, mesmo com a utilização de pressupostos controversos, como o ajuste rápido de preços (mercados se equilibram continuamente), ineficácia da PM, microfundamentados através da utilização de agente representativo e ausência de moeda (MACCALLUM, 1999).

Neste mesmo período, desenvolvimentos da corrente nova-keynesiana, por meio de modelagens dinâmicas e microfundamentado de salários e preços rígidos, com contratos escalonados, importaram na retomada da teoria velho-keynesiana, fortemente abalada pelas críticas metodológicas e teóricas da década de 70 . A incorporação do modelo de ciclo real de negócios pela escola novo-keynesiana foi realizada mediante a flexibilização das hipóteses de concorrência perfeita e preços flexíveis, substituindo-as pela concorrência monopolística (mark-up) e rigidez de preços e salários. Segundo GOODFRIEND (2007), a proposição inicial dos modelos de ciclo real de negócios foi estudar as flutuações do produto, negligenciando o papel da moeda e da PM. Ironicamente, a incorporação de rigidez de preços permitiu utilizar tais modelos para analisar as políticas monetárias ótimas.

$\mathrm{Na}$ década de 1990, os modelos de ciclos reais de negócios passaram a ser construídos a partir de agentes representativos que são retratados para resolver problemas de otimização dinâmica, bem como de interações competitivas, em estruturas de mercados com competição monopolística, presença de algum elemento de rigidez nominal de preços e salários, e que combina a disciplina teórica com maior veracidade empírica. Tais modelos, denominados "quase CRN", são estruturais, e, portanto, imunes à "crítica de Lucas" de modelos econométricos (GOODFRIEND, 2005). 
Ademais, a análise empírica tornou-se uma importante ferramenta para a validação dos modelos estruturais e a análise quantitativa da política econômica moderna. Neste contexto, os métodos econométricos e as simulações estocásticas dos modelos estruturais tornam-se parte importante do ferramental macroeconômico. Os modelos mais recentes (DSGE - Dynamic stochastic general equilibrium) contrastam com a primeira geração de modelos do ciclo real de negócios, por objetivarem mais do que insights e descrições dos mecanismos básicos de funcionamento da economia, exigindo o realismo quantitativo. Neste novo modelo, além do choque tecnológico, as políticas fiscais e monetárias passam a influenciar as flutuações econômicas (WOODFORD, 2008).

Ressalta-se que os distúrbios reais ${ }^{8}$ continuaram a desempenhar um papel central na explicação das flutuações do produto enquanto que os efeitos da PM foram incorporados, embora os seus impactos tenham sido minimizados. Por outro lado, os modelos "quase DSGE9" demonstram que o efeito da PM sobre as variáveis reais depende da política de "feedback" do BC (regras versus discricionariedade). A PM por meio de regras torna-se um instrumento eficaz para controle da inflação operando com uma âncora nominal (a inflação), dado que o comportamento dos agentes (famílias e firmas) determina os preços relativos, enquanto que a PM é capaz de afetar apenas o nível geral de preços.

Como consequência, a PM deixa de agir sobre os agregados monetários e assume um compromisso com uma meta de inflação. Neste ponto, deve-se ressaltar que tais modelos ignoram o equilíbrio do mercado monetário, deixando de formalizar uma equação tipo LM (WOODFORD, 2008). A substituição de uma equação de equilíbrio do mercado monetário por uma regra de juros deveu-se ao trabalho de TAYLOR (1993), que foi determinante para que diversos BCs considerassem e se interessassem por uma referência de PM (MACCULLUM, 1999; GOODFRIEND, 2005).

No final da década de 1990 e início de 2000, BERNANKE e GERTLER (1999 e 2001) realizaram estudos sobre as flutuações dos preços dos ativos no regime monetário de metas de inflação, identificando que a taxa de juros de equilíbrio é pouco sensível às variações destas. Os autores apontam evidência de que não existe ganho ou perda adicional para a estabilidade de preços quando um $\mathrm{BC}$

8. Principais fontes de distúrbio real são os choques tecnológicos segundo o modelo de ciclo real de negócios, embora os modelos DSGE considerem alterações nas preferências dos agentes e as políticas econômicas.

9. Os modelos "quase DSGE" correspondem aos modelos de ciclo reais de negócios, porém com a flexibilização de hipóteses como preços flexíveis e concorrência perfeita. 
resolve adotar uma resposta para as variações dos preços de ativos, exceto quando estas afetam a previsão de inflação. Este estudo é importante na medida em que influência o papel primordial do BC na busca por controle da inflação de bens e serviços, delegando ao órgão regulador (microprudencial) o papel de acompanhar o preço dos ativos em mercados específicos. Segundo os autores:

The inflation-targeting approach gives a specific answer to the question of how central bankers should respond to asset prices: Changes in asset prices should affect monetary policy only to the extent that they affect the central bank's forecast of inflation. To a first approximation, once the predictive content of asset prices for inflation has been accounted for, there should be no additional response of monetary policy to asset-price fluctuations (BERNANKE e GETLER, 2001, p. 253, grifos nossos).

Embora, BERNANKE e GETLER (1999 e 2001) sejam contrários a manipulação da taxa de juros para estabilizar preços de ativos, eles defendem que a utilização de uma PM ótima que promova a estabilidade da inflação e do produto, como no caso da adoção de um RMI, e que contribui para a estabilidade financeira, na medida em reduz a volatilidade dos ciclos de negócios (BERNANKE, 2004). Segundo BERNANKE e GETLER (2001, p. 257): "Our own view is that the macroeconomic stability associated with inflation-targeting is likely to reduce the incidence of panic-driven financial distress that could destabilize the economy, but this question is clearly deserving of further research".

Um último ponto da resenha acerca do NCM, que antecede a crise de 2007, analisa as consequências de uma taxa de juros próxima ao limite inferior a zero. Segundo EGGERTSSON e WOODFORD (2003) um BC com credibilidade poderia continuar a influenciar as taxas de juros futuras de curto prazo, à exceção de uma forte ruptura financeira. Tal argumento parte do pressuposto de que o hiato do produto e a taxa de inflação, nos modelos novo-keynesianos, são determinados pela taxa de juros de longo prazo, bem como a orientação para frente da PM (forward policy guidance) pode influenciar as expectativas em relação às taxas de juros futuras de curto prazo, dado que estas determinam o comportamento da taxa de juros de longo prazo. Segundo EGGERTSSON e WOODFORD (2003, p. 206): "We have argued that the key to dealing with a situation in which monetary policy is constrained by the zero lower bound on short-term nominal interest rates is the skillful management of expectations regarding the future conduct of policy". 
A formalização matemática do NCM, a partir de MEYER (2001) e MACCALLUM (2002) supõe a existência de uma estrutura de competição monopolística e a presença de custos relacionados ao ajuste de preços, pode ser sintetizada em três equações básicas: uma função dinâmica IS "forward looking"; uma curva de Phillips expectacional (ou função de oferta agregada); e uma função de reação da PM com base na chamada "regra de Taylor".

$$
\begin{aligned}
& y_{t}=a_{1} y_{t-1}+a_{2} E_{t} y_{t+1}-a_{3}\left(i_{t}-E_{t} \pi_{t+1}\right)+g_{t} \\
& \pi_{t}=b_{1} y_{t}^{*}+b_{2} \pi_{t-1}+b_{3} E_{t}\left(\pi_{t+1}\right)+z_{t} \\
& i_{t}=r^{*}+c_{1} y_{t-1}^{*}+E_{t}\left(\pi_{t+1}\right)+c_{2}\left(\pi_{t-1}-\bar{\pi}\right)
\end{aligned}
$$

Onde $y_{t}$ é o produto real, também conhecido como produto atual ou corrente; $y_{t}^{*}$ é o hiato do produto, representado pela diferença entre o produto real $\left(y_{t}\right)$ e a taxa natural do produto $(\bar{y}), i$ a taxa de juros nominal, $r^{*}$ a taxa de juros real de

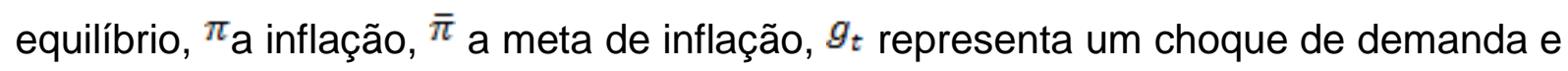
$z_{t}$ representa o choque oferta. As expectativas futuras do produto real $E_{t}\left(y_{t+1}\right)$ e da inflação $E_{t}\left(\pi_{t+1}\right)$ são tidas como expectativas racionais, enquanto que $\left(\pi_{t-1}\right)$ representam o efeito da rigidez de preços, sendo todos os coeficientes positivos. Note que as equações acima supõem um comportamento tanto "backward looking" (olhando para trás) quanto "forward looking" (olhando para frente), dada e a existência de variáveis $(\mathrm{t}-1)$ e $(\mathrm{t}+1)$. Ressalta-se, ainda, que o modelo básico é constituído por três equações (1 a 3) e três incógnitas (produto, taxa de juros e do nível de preços) a serem determinadas simultaneamente.

A equação 1 descreve uma função dinâmica IS "forward-looking", na qual o nível do produto real $\left(y_{t}\right)$ ou demanda agregada é uma função positiva do efeito defasado do produto real $\left(y_{t-1}\right)$, da renda futura esperada ${ }^{10} E_{t}\left(y_{t+1}\right)$ e do choque de demanda $\left(g_{t}\right)$, bem como é negativamente dependente da taxa de juros real de curto prazo $\left({ }^{i} t-E_{t} \pi_{t+1}\right)$. Neste modelo, o canal de transmissão da PM sobre a demanda a capacidade de usufruto permanente da riqueza existente, desconsiderando flutuações conjunturais. A renda permanente pode ser estimada através de uma média ponderada das rendas correntes e passadas das unidades detentoras de riqueza. 
agregada se processa principalmente por meio da taxa de juros e das expectativas de inflação futura. A elasticidade juros da IS $\left(a_{3}\right)$ representa a substituição intertemporal do consumo (maiores taxas de juros estimulam os agentes a postergarem consumo para o futuro), enquanto que o termo de perturbação $(g)$ sinaliza um choque de demanda que desloca a curva IS, e pode ser entendido como uma função de mudanças esperadas no gasto do governo em relação as mudanças esperadas no produto. A equação 1 sugere que as ações correntes e esperadas da PM afetam a demanda agregada. Os sinais esperados de cada termo são mostrados na forma implícita, descrita pela equação 4 abaixo:

$$
y_{t}=f\left[\begin{array}{c}
y_{t-1} E_{t} y_{t+1}\left(i_{t}-E_{t} \pi_{t+1}\right), g_{t} \\
++ \\
+
\end{array}\right]
$$

A Curva de Phillips "expectacional" ou função de oferta agregada é representada pela equação 2 , que descreve a relação direta entre a inflação $\left(\pi_{t}\right)$ e os demais termos da equação: o hiato do produto $\left(y_{t}-\bar{y}\right)$, além do efeito defasado da inflação $\left(\pi_{t-1}\right)$, das expectativas futuras de inflação $E_{t}\left(\pi_{t+1}\right)$ e do choque de oferta $\left(z_{t}\right)$. Note-se que a inflação passada é uma proxy para a rigidez de preço no curto prazo, permitindo um espaço para movimentos do produto e da inflação no curto prazo, sendo, contudo, plenamente flexível no longo prazo (hipótese de que $b_{2}+b_{3}=1 b_{2}+b_{3}=1$ ). Ademais, a expectativa de inflação futura torna-se uma variável relevante do modelo, na medida em que é incorporada enquanto uma meta intermediária da PM. Finalmente, ressalta-se que os choques de oferta $\left(z_{t}\right)$, que geram uma inflação de custos (salários e lucros), variam de modo aleatório, não são serialmente correlacionados e apresentam à média dos desvios iguais a zero, o que implica que o efeito destes sobre o nível de preços no longo prazo é igual a zero. Os sinais de cada termo da equação 2, descrito na forma implícita, são mostrados na equação abaixo.

$$
\pi_{t}=f\left[\begin{array}{c}
y_{t}>\bar{y} \\
+\begin{array}{c}
\pi_{t-1} \\
+
\end{array} E_{t}\left(\pi_{t+1}\right) ; \\
+
\end{array}\right]
$$

Na equação 3 é apresentada uma função que descreve a regra da PM (regra de Taylor), e relaciona positivamente a taxa de juros nominal de curto prazo (o instrumento de PM) com a taxa natural de juros real $\left(r^{*}\right)$, o hiato do produto defasado 
$\left(y_{t-1-} \bar{y}\right)$, as expectativas futuras de inflação $\left(E t\left(\Pi_{t+1}\right)\right)$ e a taxa de inflação passada $\left(\pi_{t-1}\right)$. Desta forma, a PM responde aos desvios do produto e da inflação, dado os objetivos de estabilidade de preços e do produto. Como resultado o modelo do NCM implica que a PM é conduzida para estabelecer uma meta para a taxa básica de juros sem qualquer consideração sobre a taxa de crescimento da moeda. Os sinais esperados de cada termo são mostrados na equação 6 a seguir.

$i_{t}=f\left[\begin{array}{cc}r^{*} & y_{t-1}>\bar{y}_{t} E_{t}\left(\pi_{t+1}\right) ; \\ + & \left(\pi_{t-1}>\bar{\pi}\right) \\ + & +\end{array}\right]$

Nesta equação observa-se que a curva LM é substituída pela regra de PM, dado que no modelo a equação de equilíbrio no mercado monetário não é necessária para a determinação da produção, dos preços e da taxa de juros, tornando a oferta de moeda uma variável endógena e residual. No entanto, esta hipótese depende da estabilidade da demanda por moeda para que a relação entre esta e a inflação seja estável no longo prazo.

Uma segunda hipótese da regra de PM tem como implicação que a taxa de juros de curto prazo, definida pelas operações de mercado aberto, é a principal referência para os preços da economia, de modo que a taxa de juros de longo prazo, os preços dos ativos e da taxa de câmbio deverão se mover de forma estável e previsível em resposta as variações nas taxas de PM. Esta premissa representa um entrave do NCM, além de apresentar problemas para a condução da PM, quando a taxa de juros de curto prazo encontra-se próxima ao limite inferior igual a zero (ou em uma situação de armadilha de liquidez, como observada na crise americana de 2007-2008).

Esta questão, segundo MACCALLUM (1999), está diretamente relacionada à ausência de uma equação de demanda por moeda e poderia se transformar em uma limitação da teoria para uma situação na qual a taxa de juros de curto prazo aproxima-se de zero, devido à simplificação do modelo em relação aos mecanismos de transmissão da PM. Contudo não representava nenhum entrave, pois era amplamente aceita, até a crise de 2007-08, a proposição de que nesta circunstância o BC poderia continuar a conduzir a PM através da orientação para a frente da PM. 
O intenso debate do mainstream nas últimas décadas resultou na construção de uma nova síntese neoclássica, denominada de NCM. O resultado foi um novo regime monetário, denominado de RMI. Este está diretamente associado à necessidade de se definir uma nova âncora nominal (inflação) para a PM, resultante do fim do regime de câmbio fixo do início da década de 1970 (Bretton Woods), além das dificuldades encontradas na adoção de metas monetárias, em virtude das inovações financeiras e das variações, não previsíveis, na velocidade de circulação da moeda. Ademais, as mudanças foram impulsionadas pelas experiências de condução de política adota pelas autoridades monetárias em diversos países. Segundo MACCALLUM (1999, p. 13):

One fact is that several (regional) Federal Reserve Banks have, since the late 1970s, employed academic economists as consultants, a practice that makes each group more familiar with research assumptions held to be essential by the other - e.g., academics have become more knowledgeable about realistic operating procedures while central bank economists have become more comfortable with analysis utilizing rational expectations.

No RMI, BERNANKE e MISHKIN (1997) argumentam que embora existam diferenças na sua aplicação em diversos países, este pode ser mais bem compreendido como uma estratégia de condução da PM baseada em regras, reforçando a coerência e a transparência da PM. Neste regime, a autoridade monetária define explicitamente como objetivo principal, senão único da PM o cumprimento, ao longo de um determinado período, de uma meta de inflação, podendo ser uma banda ou um determinado valor alvo com intervalo de tolerância.

A estabilidade de preços torna-se o objetivo final da PM para um horizonte de médio e longo prazo, respaldado na hipótese de inexistência de trade off de longo prazo (neutralidade da moeda) que torna inócuo qualquer tentativa da autoridade monetária de explorar a curva de Phillips. Entretanto, a existência de trade-off de curto prazo, como decorrência da rigidez de preços e salários, abre um espaço para a utilização da demanda agregada, via PM que promova estabilização de preços, buscando minimizar o impacto do desvio do produto em relação a sua taxa natural e consequentemente sobre a inflação. Segundo BERNANKE e MISHKIN (1997, p. 100) "The rationale for treating inflation as the primary goal of monetary policy is clearly strongest when medium- to long-term horizons are considered, as most economists agree that monetary policy can affect real quantities, such as output and employment, only in the short run". 
Ao tornar a taxa de inflação a ancora do RMI, a autoridade monetária reduz significativamente o papel das metas intermediárias (taxa de câmbio e dos agregados monetários) em detrimento da taxa de juros de longo prazo e da manipulação das expectativas de inflação, enquanto objeto de estabilização de curto prazo, uma vez que estas podem se tornar inconsistentes com as metas de inflação, em caso de conflito (BERNANKE e MISHKIN, 1997). Neste regime, a prioridade do $\mathrm{BC}$ é reduzir a volatilidade e manter a inflação baixa, delegando, normalmente, um papel secundário para 0 amortecimento das flutuações cíclicas do produto (MACCALLUM, 2005, p. 289).

Nesta perspectiva, a consecução do objetivo do BC deverá ajustar o instrumento de PM (taxa de juros nominal corrente) para que a inflação corrente convirja em direção à meta de inflação de longo prazo previamente estabelecida. No entanto, isso só será possível de se alcançar em um prazo curto (imediatamente), na ausência de inflação (choques) de custo ou despreocupação com o desvio da produção. Neste ponto, existem divergências em relação à adoção de uma meta extremada (convergência imediata para a meta de longo prazo), ou meta gradual (convergência ao longo do tempo), embora a segunda seja predominante nas práticas observadas pelos BCs (CLARIDA et. al., 1999).

O instrumento de PM, a partir de uma regra de Taylor, deve subir/reduzir para afetar a taxa futura de juros de curto prazo e deste modo a taxa de juros de longo prazo e as expectativas de inflação, promovendo a contração ou expansão da demanda agregada, dependendo do comportamento do nível de preços. No entanto, a autoridade monetária deve ser capaz de identificar as fontes de choques (temporárias) no ciclo econômico, de modo a ajustar a taxa futura de juros de longo prazo, através da taxa nominal de curto prazo para os choques de demanda, porém a taxa nominal deve permanecer constante quando ocorrerem choques de oferta ${ }^{11}$. (CLARIDA et al.,1999). Caso contrário, o BC enfrentará um trade off de curto prazo entre estabilizar a inflação ou o produto (BERNANKE, 2004).

No RMI, são criados mecanismos institucionais que tornam o BC responsável pelo controle inflacionário, devendo este atuar de forma transparente, divulgando a meta numérica para a inflação e suas intenções sobre a política de juros e atuando de forma persistente para resolver os choques inflacionários, minimizando os impactos sobre o produto (GOODFRIEND, 2007). Periodicamente, os BCs divulgam

11. Suposição de que a inflação de custos varia de modo aleatório, não são serialmente correlacionados e apresentam a média dos desvios iguais à zero, o que implica que o efeito da inflação de custos no longo prazo é igual a zero. 
relatórios detalhados sobre as suas avaliações de inflação e conjuntura econômica. O compromisso consistente com a estabilidade de preços mostrou-se viável para reduzir a volatilidade e levar a inflação a um patamar baixo (GOODFRIEND, 2005, p. 252). Estes princípios estão de acordo com a proposição teórica de credibilidade e transparência da autoridade monetária e o problema de inconsistência temporal, convergindo para a tese de independência do BC.

$\mathrm{Na}$ figura 1 sintetiza-se a discussão desta seção, enfatizando as hipóteses, modelos, metodologia e os elementos operacionais do RMI. Conforme abordado, as principais contribuições teóricas são oriundas das escolas novo clássica e de ciclo reais de negócios que foram alteradas para incorporar a rigidez de preços no curto prazo, além da concorrência imperfeita, pelos novos-keynesianos. A partir destes pressupostos e da metodologia de equilíbrio geral estocástico, o modelo ganha a forma de uma curva de Phillips e uma função IS. A operacionalidade da PM é representada pela regra de Taylor, a inflação enquanto a ancora nominal e a taxa de juros e a comunicação como as metas operacionais, que são passiveis de alterar as metas intermediárias (taxa de juros de longo prazo e expectativas de inflação) e deste modo à demanda agregada e consequentemente a inflação.

Figura 1. Aspectos teóricos, metodológicos e operações do RMI

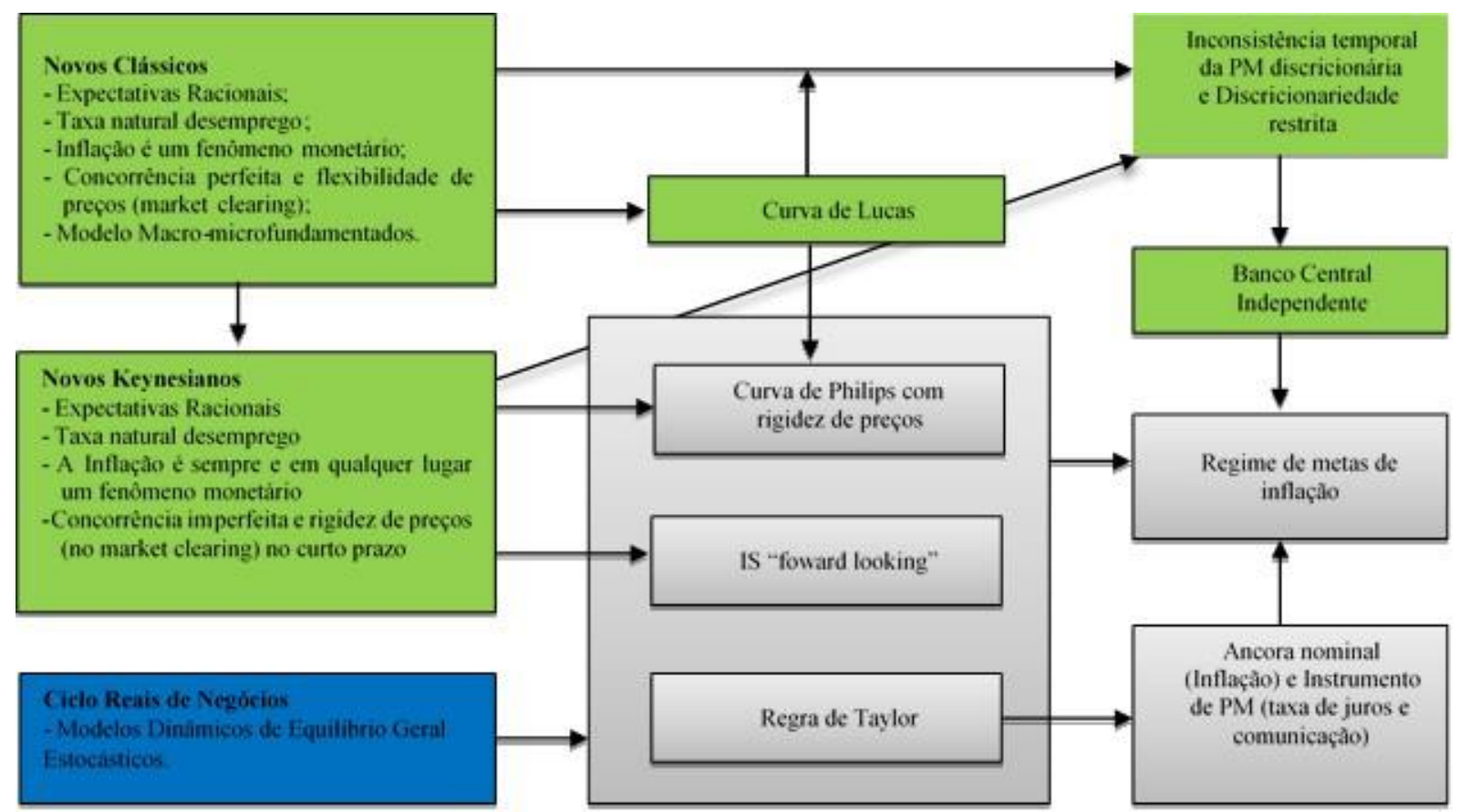

Fonte: Elaboração própria 


\section{CRISE, REAFIRMAÇÃO DO NCM E A REVISÃO DO RMI}

A ruptura no sistema financeiro americano, iniciada em setembro de 2007 e agravada a partir de setembro de 2008, promoveu uma crise sistêmica que impactou sobremaneira a economia mundial. Tecnicamente a recessão americana começou em dezembro de 2007 e se estendeu até junho de 2009, embora os indicadores econômicos para esta economia ainda permaneçam abaixo do período que antecede a crise. Registrou-se para este país um crescimento econômico entre 2007 e 2014 de 1,2\% a.a., ao passo que a inflação média foi 1,7\% a.a. No mercado de trabalho, observaram-se resultados ruins com a taxa de desemprego subindo de $5 \%$ em 2006 para cerca de 10\% em 2009, 7,4\% em 2013 e fechando o ano de 2014 em $6,2 \%$.

Além dos EUA a crise teve fortes repercussões sobre a economia mundial, tendo sido nominada pelo Bank for International Settlements (BIS) de a "grande recessão de 2008/2009". Tal fato se deve a redução significativa na taxa de crescimento que caiu de 4,7\% na média do período de 2003 a 2007, para 3\% em 2008, -0,3\% em 2009 e fechando o ano de 2013 em 2,4\%. Observou-se que choques negativos foram mais significativos nas economias desenvolvidas, na qual a maior integração e diversificação de seus mercados financeiros promoveram perdas substanciais em diversos ativos, além de forte desaceleração do produto e do emprego. No caso das economias em desenvolvimento, o impacto sobre os mercados financeiros deveu-se a volatilidade do fluxo de capitais e a queda na produção (e comércio) que derivou da redução na demanda agregada mundial por bens e serviços.

Deste modo, a crise recente demonstrou as fraquezas dos argumentos de que a PM adotada ao longo das últimas décadas promovera uma "grande moderação", bem como demonstrou que o ambiente econômico, relativamente estável, não protege a economia da instabilidade financeira e de seus efeitos sobre a atividade econômica. Ao invés disso a estabilidade de preços e do produto e as baixas taxas de juros promoveram a percepção generalizada da redução dos prêmios de risco a patamares muito reduzidos, ampliando significativamente a concessão de crédito e tornando o sistema financeiro vulnerável (MINSHKIN, 2011 e BLANCHARD et al., 2013). Esta situação foi descrita por BORIO e ZHU (2008) como o canal de assunção ao risco. 
A ruptura financeira na economia americana demonstrou o quanto o ambiente macroeconômico poderia ser não linear, e que os impactos significativos sobre o nível da atividade econômica poderiam perdurar por um longo período. Neste contexto de crise, a taxa de juros perde rapidamente a sua eficácia, quando se aproxima do limite inferior igual a zero, e as políticas não convencionais, monetárias e fiscais, são acionadas para evitar que a recessão se torne um processo circular. Ademais, observou-se o impacto da crise sobre a estabilidade fiscal, promovendo aumento do endividamento público (MINSHKIN, 2012; BLANCHARD et al., 2013; e REINHART e ROGOFF, 2009).

It was tempting for macroeconomists and policymakers alike to take much of the credit for the steady decrease in cyclical fluctuations from the early 1980s on and to conclude that we knew how to conduct macroeconomic policy. We did not resist temptation. The crisis clearly forces us to question our earlier assessment. (BLANCHARD et al., 2010, p. 3)

Entretanto, em meio as desordens causadas pela crise, os teóricos do NCM, em particular BLANCHARD et al. (2010 e 2013), BERNANKE (2009, 2012a, 2012b), BLINDER (2010), YELLEN (2011a e 2011b), MINSHKIN (2010, 2011, 2012) e WOODFORD (2012a e 2012b), vêm reafirmando que a crise e as medidas não convencionais adotadas para conter o pânico nos mercados financeiros, além do forte desaquecimento da atividade econômica, não abalaram a estrutura teórica, prevalecendo os princípios da NCM, quais sejam:

1) inflation is always and everywhere a monetary phenomenon; 2) price stability has important benefits; 3 ) there is no long-run tradeoff between unemployment and inflation; 4) expectations play a crucial role in the determination of inflation and in the transmission of monetary policy to the macroeconomy; 5) real interest rates need to rise with higher inflation, i.e., the Taylor Principle; 6) monetary policy is subject to the time-inconsistency problem; 7) central bank independence helps improve the efficiency of monetary policy; 8) commitment to a strong nominal anchor is central to producing good monetary policy outcomes; and 9) financial frictions play an important role in business cycles $^{12}$. (MINSHKIN, 2011, p. 3).

Esta avaliação sugere que a essência dos elementos da convergência teórica pré-crise deverá permanecer, conforme abordado por MISHKIN (2011, p.32), que afirma:

The monetary policy strategy that follows from the eight principles of the new neoclassical synthesis is what I have referred to, for want of a better name, as flexible inflation targeting. Since, as I have argued here, none of these principles is invalidated by the events of the recent financial crisis, this approach to monetary policy strategy is still equally valid. The arguments

12. O item 9 já havia sido apresentado por MINSHKIN (2007), no entanto não era amplamente aceita pelo NCM. 
supporting central banks' adhering to the principles of the new neoclassical synthesis are still every bit as strong as they were before the crisis.

Esta visão também é compartilhada por BLANCHARD et al. (2010, p. 10)

It is important to start by stating the obvious, namely, that the baby should not be thrown out with the bathwater. Most of the elements of the precrisis consensus, including the major conclusions from macroeconomic theory, still hold. Among them, the ultimate targets remain output and inflation stability. The natural rate hypothesis holds, at least to a good enough approximation, and policymakers should not assume that there is a long-term trade-off between inflation and unemployment. Stable inflation must remain one of the major goals of monetary policy. Fiscal sustainability is of the essence, not only for the long term, but also in affecting expectations in the short term.

De modo similar aos demais autores WOODFORD (2012b, p.26-27) assume que:

that inflation targeting frameworks can and should be adapted to take account of the possibility of intermittent disruptions of financial intermediation of the kind experienced in 2007-2009. (...) Both the possibility of sometimes hitting the zero lower bound and the possibility of sometimes needing to use interest-rate policy to restrain the growth of risks to financial stability make these reforms of inflation targeting practice all the more urgent. But the reforms that are needed are a natural. But the reforms that are needed are a natural extension of the logic of inflation - forecast targeting, rather than a repudiation of its central aims.

Contudo, mesmo que a crise e as PMNC não tenham abalado à estrutura teórica do NCM, pelo menos na visão destes autores, ela expos vulnerabilidades e limitações da PM que vem sendo repensadas pelos fundadores do NCM e pelos BCs na condução do RMI. A convergência, refere-se a transitoriedade das PMNC e a necessidade de que estas políticas de estímulos monetários sejam retiradas da economia. De outro modo ela gerou uma forte divergência entre os diversos pesquisadores a propósito de como deverá ser conduzida a PM pós-crise.

Os desacordos, entre os autores, distinguem-se em relação às proposições aditivas ao PM, tais como uma maior flexibilização do RMI, representadas pela necessidade de a PM interagir com as políticas macroprudenciais, controle de capitais e câmbio, assim como a redefinição do papel da política fiscal. Nesta perspectiva YELLEN (2014) defende a flexibilização do RMI, através da introdução de novos objetivos e instrumentos. Segundo MISHKIN (2012, p.11): "In other words, the rationale for flexible inflation targeting is every bit as strong as it was before the crisis. However, the key facts learned from the crisis do suggest that what is meant by flexibility in an inflation targeting regime need to be rethought".

No que se refere ao debate em relação à condução da PM, embora a inflação permaneça como ancora nominal e o objetivo central da política econômica, quatro 
proposições vêm sendo debatidas. A primeira refere-se a proposta de BLANCHARD et al (2010) de aumentar o valor da meta de inflação, permitindo maior efetividade para a taxa de juros até o limite zero, sendo este ponto questionado por MINSHKIN (2011) em virtude da dificuldade de se ancorar as expectativas de inflação quando os valores desta são mais altos. Um segundo ponto que vem apresentando convergência de propostas refere-se à efetividade da PM não convencionais para as situações de armadilha da liquidez. Contudo a utilização de PM para lidar com situações de bolhas permanece um tema polémico, sendo defendido por MINSHKIN (2011) e WOODFORD (2012a) em relação ao gerenciamento de risco (utilização de taxa de juros para estourar bolhas) e questionado por BLANCHARD (2013) que identifica maior efetividade na adoção de políticas financeiras. Ressalta-se ainda YELLEN (2014) que vem defendendo novos objetivos (inflação, produto, estabilidade financeira) a introdução de novos instrumentos (taxa de juros, comunicação, reservas, compra de ativos de longo prazo etc.).

No que se refere a esta última questão, diversos autores (MISHKIN, (2011), BERNANKE (2012a), BLANCHARD et al. (2010 e 2013) e WOODFORD (2012a e 2012b)) vêm defendendo a inclusão de um novo instrumento (políticas macroprudenciais) e um novo objetivo (estabilidade financeira) ao arcabouço da PM, devendo este ser coordenado pelo BC. Nesta perspectiva a revisão do NCM suprimiu a ideia dicotômica entre a PM e a política financeira, sendo que os dois instrumentos devem operar de forma conjunta, desempenhando as funções de estabilização inflacionária, do produto e financeira. Segundo BAYOUMI et al (2014), a PM incorporação de alguns novos instrumentos acessórios de política tendo em vista a necessidade de conjugação do objetivo central de política econômica (estabilidade de preços) com outros objetivos intermediários (como estabilidade financeira), evitando assim a visão simplista de "um objetivo um instrumento" que caracterizou o período anterior à crise americana.

No que se refere ao controle de capitais e gerenciamento de câmbio, embora seja um dos temas mais divergentes da revisão do NCM, observou a partir da recente crise a excessiva vulnerabilidade das economias emergentes em decorrência das mudanças abruptas no fluxo internacional de capitais. Como resultado, BLANCHARD et al (2010), DE GREGORIO (2011) e SUBBARAO (2014) tem defendido a utilização de controle de capitais, ainda que em períodos de excepcionalidade. Em relação ao gerenciamento câmbio, OSTRY et al. (2012) defendem que as economias emergentes incorporem a meta de taxa de câmbio real 
e o instrumento de intervenção cambial, enquanto um segundo componente de um RMI, para lidar com os efeitos adversos do fluxo de capitais sobre a estabilidade macro e financeira.

Finalmente BLANCHARD et al. (2010 e 2013) defende que a política fiscal, embora subjugada aos objetivos de estabilidade de preços da PM, deve desempenhar a função de estabilizador automático, promovendo o "espaço fiscal" em períodos de crescimento econômico que permitam utilizar política fiscal em situações de exceção, contudo o objetivo de longo prazo deve ser o equilíbrio orçamentário. Ressalta-se que tal proposição é questionada por MISHKIN (2012) que argumenta que a política fiscal pós-crise deverá promover uma redução do nível de endividamento e manter a função de sustentabilidade do orçamento governamental.

$\mathrm{Na}$ figura 2, buscou-se retratar, de uma forma bem geral, os principais pontos de discussões em relação a flexibilização do RMI, apontando as proposições de mudança, que embora não sejam consensuais, representam a visão de diversos autores.

Figura 2. Aspectos teóricos, metodológicos e operações da revisão do RMI

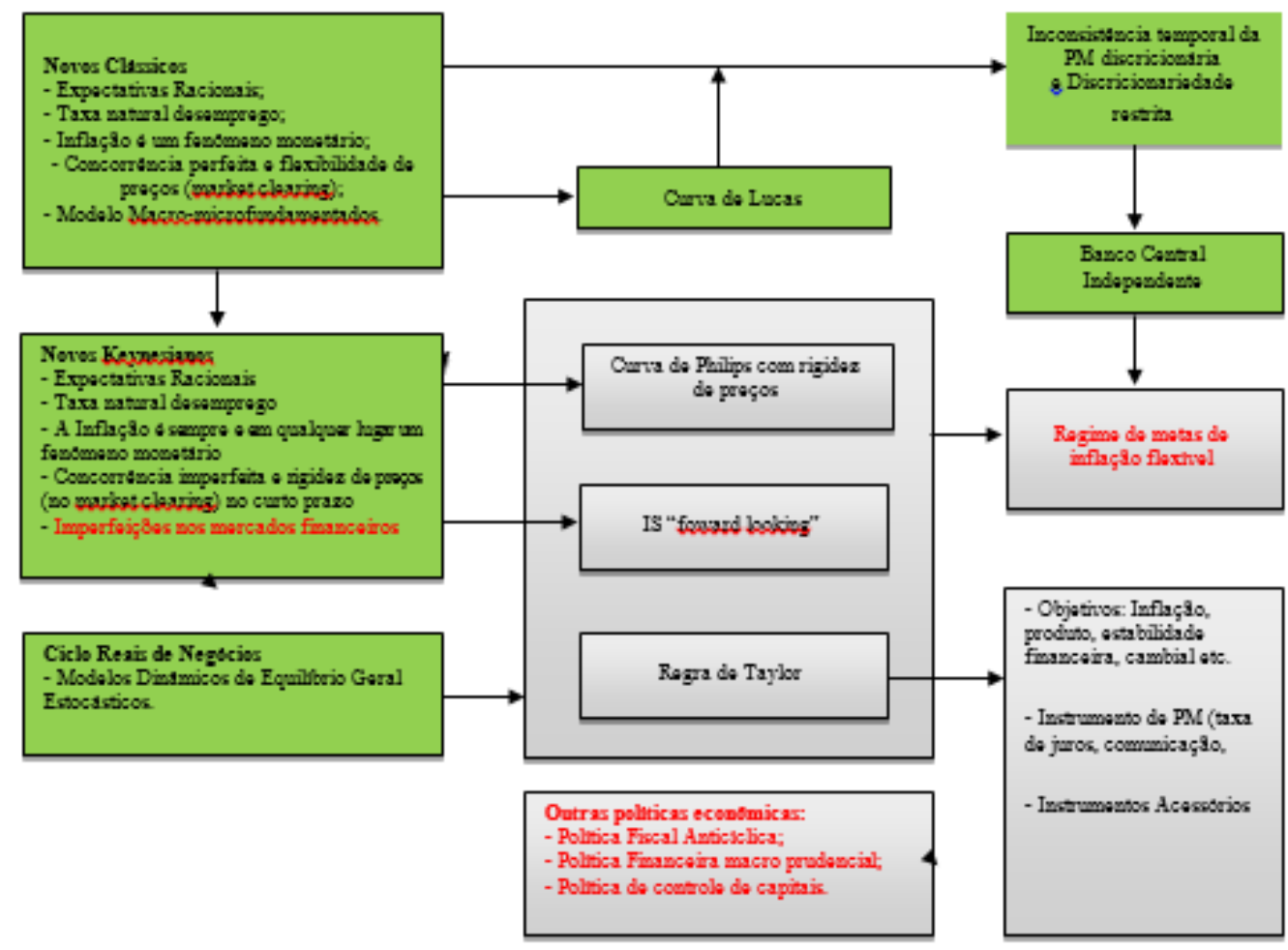

Fonte: Elaboração própria. 


\section{CONSIDERAÇÕES FINAIS}

A revisão do NCM mantém a essência teórica do NCM. Evidencia-se a manutenção da "inflação enquanto um fenômeno monetário", das hipóteses de expectativas racionais, taxa natural de desemprego e a rigidez de preços no curto prazo, além de suas implicações para os modelos de inconsistência temporal e de um BC Independente. Contudo, as mudanças mais significativas podem ser observadas em relação à condução da política econômica, por meio de uma flexibilização do RMI.

A crise financeira foi entendida pelo NCM como a ocorrência de falhas dos mercados financeiros, além da ausência de política financeira (macro prudencial), até então ignorada pela aceitação da hipótese de mercados eficientes ("doutrina Greenspan"). Senão de outro modo, emergiram divergências dentro do mainstream, cujos resultados, ainda que prematuros, apontam para a incorporação de gerenciamento de risco aos objetivos intermediários da PM e as políticas financeiras (macroprudenciais) como um novo instrumento de PM. Nesta perspectiva, embora uma nova meta operacional e instrumento de PM sejam propostos, estes não alteram a essência do RMI, uma vez que a inflação permanece como a meta final de política e a taxa de juros de curto prazo e a comunicação os instrumentos operacionais.

Ademais se observou a importância da política fiscal anticíclica e o problema da necessidade de se ter espaço fiscal nas economias desenvolvidas e a interrelação entre a PM das economias centrais e não centrais, evidenciando os efeitos da taxa de juros e incertezas sobre o fluxo de capitais e deste nas variações cambiais. Em síntese, a discussão ortodoxa pós-crise, mantém os fundamentos do NCM e flexibiliza o RMI, delegando novas funções, ainda que subordinadas, as políticas financeiras, fiscais e cambiais.

\section{REFERÊNCIAS}

BAYOUMI, T, DELL'ARICCIA, G., HABERMEIER, K., MANCINI-GRIFFOLI, T, e VALENCIA, F. Monetary Policy in the New Normal. IMF Staff Discussion Note SDN 14/3, abril de 2014.

BERNANKE, B. The Great Moderation. Remarks before the Eastern Economic

Association, Washington, DC, 20 de fevereiro de 2004. 
BERNANKE, B. The Crisis and the Policy Response. Stamp Lecture, London School of Economics, London, England, janeiro de 2009.

BERNANKE B. Some reflections on the crisis and the policy response. The Century Foundation Conference on "Rethinking Finance", New York, 13 de Abril de 2012a.

BERNANKE, B. Monetary Policy since the Onset of the Crisis. The Federal Reserve Bank de Kansas City Economic Symposium, Jackson Hole, Wyoming, 31 de Agosto de 2012b. BERNANKE, B.; GERTLER, M. Monetary Policy and Asset Volatility. Federal Reserve Bank of Kansas City Economic Review, v. 84, n.4, p. 17-52, 1999.

BERNANKE, B.; GERTLER, M. Should Central Banks Respond to Movements in Asset Prices? The American Economic Review, v. 91, n. 2, p. 253-257, 2001.

BLANCHARD, O. There a Core of Usable Macroeconomics? The American Economic Review, v. 87, n. 2, p. 244-246, 1997.

BLANCHARD, O.; DELL'ARICCIA, M.; MAURO, P. Rethinking Macroeconomic Policy. Journal of Money, Credit and Banking, v. 42, n. 6, p.199-215, 2010.

BLANCHARD, O.; DELL'ARICCIA, M.; MAURO, P. Rethinking Macro Policy II: Getting Granular. IMF Staff Discussion Notes No. 13/03, p. 199-215, 2013.

BLANCHARD, O.; KATZ, L. What We Know and Do Not Know about the Natural Rate of Unemployment. Journal of Economic Perspectives, v.11, n.1, p. 51-72, 1997.

BLINDER, A. Quantitative Easing: Entrance and Exit Strategies. Federal Reserve Bank of St. Louis Review, v. 92, n.6, p. 465-79, 2010.

BORIO, C.; ZHU, H. Capital regulation, risk-taking and monetary policy: A missing link in the transmission mechanism? BIS Working Papers n. 268, dezembro de 2008.

CLARIDA R.; GALÍ J.; GERTLER, M. The Science of Monetary Policy: A New Keynesian Perspective. Journal of Economic Literature, Vol. XXXVII, p. 1661-1707, 1999.

DE GREGÓRIO, J. Los flujos de capital y la interacción entre la política macroprudencial y la política monetaria. Conferência Anual del Banco Central de Chile, La Movilidad de Capitales y la Política Monetaria, Santiago, 17-18 de novembro de 2011.

DE GREGORIO, J. Capital flows and capital account management. In: G. Akerlof, O. Blanchard, D. Romer e J. Stiglitz. What Have We Learned? Macroeconomic Policy after the Crisis. Cambridge, MA: The MIT Press, 2014.

EGGERTSSON, G.; WOODFORD, M. The Zero Bound on Interest Rates and Optimal Monetary Policy. Brookings Papers on Economic Activity, v.34, p.139-211, 2003.

ESTRELLA, A.; MISHKIN, F. S. Rethinking the Role of NAIRU in Monetary Policy: Implications of Model Formulation and Uncertainty. In TAYLOR, J. (Org.), Monetary Policy Rules. Chicago: University of Chicago Press, 1999.

FRIEDMAN, M. The quantity theory of money - a restatement. In FRIEDMAN, M. (Org.)

Studies in the quantity theory of money. Chicago: Chicago University Press, 1956.

FRIEDMAN, M. The Role of Monetary Policy, The American Economic Review, v. LVIII, n. $1,1968$.

GOODFRIEND, M. Monetary Policy in the New Neoclassical Synthesis: a Primer.

Economic Quarterly Federal Reserve Bank of Richmond, v. 90/3, p.21-45, 2004. 
GOODFRIEND, M. The Monetary Policy Debate since October 1979: Lessons for Theory and Practice. Federal Reserve Bank of St. Louis Review, v. 87, n. 2, pp. 243-62, 2005.

GOODFRIEND, M. How the World Achieved Consensus on Monetary Policy. Journal of Economic Perspectives, v. 21, n. 4, p. 47-68, 2007.

GOODFRIEND, M.; KING, R. The New Neoclassical Synthesis and the Role of Monetary Policy. In BERNANKE, B. ROTEMBERG, J. (Org.). NBER Macroeconomics Annual, junho de 1997.

GOODFRIEND, M. Lessons Learned from a Century of Federal Reserve Last Resort Lending. Testimony before the Subcommittee on Monetary Policy and Trade, U.S. House of Representatives. Washington, 11 de setembro de 2013.

LUCAS, R. Econometric policy evaluation: a critique. Carnegie-Rochester Conference Series on Public Policy, v. 1, n. 1, p.19-46, 1976.

MCCALLUM, B. Recent developments in monetary policy analysis: the role of theory and evidence. NBER Working Paper n. 7088. Cambridge, MA, abril de 1999.

MCCALLUM, B. What Have We Learned Since October 1979? Federal Reserve Bank of St. Louis Review, Part 2, p.287-291, março/abril 2005.

MEYER. L. Does Money Matter? Federal Reserve Bank of St. Louis, p.1-15, set./out. 2001.

MISHKIN, F. Will Monetary Become More of a Science? NBER Working Paper, Cambridge: National Bureau of Economic Research, n. 13566, outubro de 2007.

MISHKIN, F. S. Monetary Policy Strategy: Lessons from the Crisis. National Bureau of Economic Research. Working Paper 16755, fevereiro de 2011.

MISHKIN, F. S. Central Banking After the Crisis. Prepared for the 16th Annual Conference of the Central Bank of Chile, Santiago, Chile, 15-16 de novembro de 2012.

OSTRY, D; GHOSH, R.; CHAMON; M. Two Targets, Two Instruments: Monetary and Exchange Rate Policies in Emerging Market Economies. Staff Discussion Notes No. 12/01, fevereiro de 2012.

PAULA, L.F.; SARAIVA, P.; MODENESI, A. "Revisão pós-crise do Novo Consenso Macroeconômico: mais do mesmo?". Revista da Sociedade Brasileira de Economia Política v. 50, p. 38-64, 2018.

REINHART, C. e ROGOFF, K. This Time Is Different: Eight Centuries of Financial Folly, Princeton University Press, Princeton, NJ, 2009.

ROGOFF, K. The Optimal Degree of Commitment to an Intermediate Monetary Target. Quartely Journal of Economics, v.100, p. 1169-89, 1985.

SARAIVA, P., PAULA, L.F.; MODENESI, A. Crise americana e as políticas monetárias nãoconvencionais. Economia e Sociedade, v. 26, n.1, p.1-44, 2017.

SUBBARAO, D. (2014). Capital account management: toward a new consensus? In: Akerlof, G.; Blanchard, O.; Romer, D.; Stiglitz, J. (Org.). What Have We Learned? Macroeconomic Policy after the Crisis. Cambridge, MA: The MIT Press, 2014. 
TAYLOR, J. Discretion versus Policy Rules in Practice. Carnegie-Rocherter Conference Series on Public Policy 39, p. 195-2014, 1993.

TAYLOR, J. Core of practical macroeconomics. The American Economic Review, v. 87, n. 2, p. 233-235, 1997.

TAYLOR, J. Does the Crisis Experience Call for a New Paradigm in Monetary Policy? CASE Network Studies and Analyses n. 402/2010. Warsaw: CASE-Center for Social and Economic Research, 2010.

TAYLOR, J. Remarks at a Panel on the Monetary Policy Implications of the Global Crisis. International Journal of Central Banking Conference Hosted by the Bank of Japan. Tokyo: Bank of Japan, 16-17 de setembro de 2010.

WOODFORD, M. Convergence in Macroeconomics: Elements of the New Synthesis. American Economic Journal, v. 1, p. 267-279. 2008.

WOODFORD, M. Methods of Policy Accommodation at the Interest-Rate Lower Bound. Proceedings - Economic Policy Symposium - Jackson Hole, Federal Reserve Bank of Kansas City, p. 185-287, 2012a.

WOODFORD, M. Inflation Targeting and Financial Stability. Sveriges Riks Bank Economic Review, 2002-1, p.7-32, 2012b.

YELLEN, J. Unconventional monetary policy and central bank communications, Speech at The University of Chicago Booth School of Business US Monetary Policy Forum, New York, fevereiro de 2011a.

YELLEN, J. The Federal Reserve's Asset Purchase Program. Speech at the Brimmer Policy Forum, Allied Social Science Associations Annual Meeting. Denver, Colorado 8 de janeiro de 2011b.

YELLEN, J. Many Targets, Many Instruments: Where Do We Stand? In: Akerlof, G.; Blanchard, O.; Romer, D.; Stiglitz, J. (Org.). What Have We Learned? Macroeconomic Policy after the Crisis. Cambridge, MA: The MIT Press, 2014.

\section{NOTAS DE AUTOR}

\section{CONTRIBUIÇÃO DE AUTORIA}

Paulo Saraiva - Concepção. Coleta de dados, Análise de dados, Elaboração do manuscrito, revisão e aprovação da versão final do trabalho

Luiz Fernando de Paula - Concepção e elaboração do manuscrito. Coleta de dados Participação ativa da discussão dos resultados; Revisão e aprovação da versão final do trabalho.

André de Melo Modenesi- Concepção e elaboração do manuscrito. Coleta de dados Participação ativa da discussão dos resultados; Revisão e aprovação da versão final do trabalho.

FINANCIAMENTO

Não se aplica.

CONSENTIMENTO DE USO DE IMAGEM

Não se aplica.

APROVAÇÃO DE COMITÊ DE ÉTICA EM PESQUISA

Não se aplica.

CONFLITO DE INTERESSES

Não se aplica.

Este artigo está licenciado sob a Licença Creative Commons CC-BY. Com essa licença você pode compartilhar, 
adaptar, criar para qualquer fim, desde que atribua a autoria da obra.

HISTÓRICO - uso exclusivo da revista

Recebido em: 26-12-2018

Aprovado em: 25-01-2019 